\title{
Nanomechanics of Nonideal Single- and Double-Walled Carbon Nanotubes
}

\author{
C. H. Wong and V. Vijayaraghavan \\ School of Mechanical and Aerospace Engineering, Nanyang Technological University, 50 Nanyang Avenue, Singapore 639798
}

Correspondence should be addressed to C. H. Wong, chwong@ntu.edu.sg

Received 24 March 2012; Accepted 12 June 2012

Academic Editor: Leonard Deepak Francis

Copyright ( $) 2012$ C. H. Wong and V. Vijayaraghavan. This is an open access article distributed under the Creative Commons Attribution License, which permits unrestricted use, distribution, and reproduction in any medium, provided the original work is properly cited.

\begin{abstract}
The buckling characteristics of nonideal single- and double-walled carbon nanotubes were studied in this work via molecular dynamics simulation method. An imperfectly straight nonideal single-walled carbon nanotube (SWCNT) with a bent along the tube axis was used to form an array which is subjected to compression. The change in orientation of bends will result in a variation of nonbonded interactions in an SWCNT array system. We find that these variations in the nonbonded interactions strongly affect the buckling resistance of the SWCNT array. Similarly, a nonideal double-walled carbon nanotube (DWCNT) is constructed by varying the interlayer distance by introducing a center offset on the inner core SWCNT. The inclusion of offset along the tube axis in such nonideal DWCNT can enhance or deteriorate the mechanical qualities of the DWCNT under compression. Our numerical studies on nonideal CNT systems suggest a possibility of designing high-performing CNTs for applications involving fiber reinforcements.
\end{abstract}

\section{Introduction}

Carbon nanotubes (CNTs) have attracted a significant research interest in the academic and industry circles due to its remarkable characteristics [1]. Extensive application of CNTs has been in the field of structural engineering due to its high tensile strength [2-4]. Most investigations on the mechanical characteristics of CNTs have been performed theoretically by deploying continuum mechanics or molecular dynamics (MD) simulation technique. The release in strain energy of CNTs subjected to large deformations has been explained by making use of a continuum shell model proposed by Yacobson et al. [5]. The variation of the buckling load with the CNT diameter has been analyzed by Liew et al. [6]. The REBO potential was further modified to include the long-range effects to study the effect of van der Waal forces on the compressive strength of CNT by Che et al. [7]. Ru [8] developed an elastic double-shell model to describe the infinitesimal buckling of a doublewalled carbon nanotube (DWCNT). A systematic analysis on the buckling of multiwalled CNT (MWCNT) under radial pressure was investigated by Wang et al. [9]. They introduced an approximate method to replace MWCNT by few layered elastic shell. The effect of boundary conditions on the buckling behavior of MWCNTs has been studied by Tong et al. [10]. They found that clamping the outermost CNT in an MWCNT can enhance the critical strain up to four times the original value. The buckling of DWCNTs subjected to torsion or compression has been studied by Lu et al. [11]. They developed a continuum model to account for the van der Waals interactions that act in between the layers of the DWCNT. The effect of tube radius on the critical buckling load of a MWCNT has been investigated by $\mathrm{He}$ et al. [12]. They derived explicit formulas to describe the interlayer van der Waals interaction in an MWCNT. A molecular structural mechanics approach was utilized to explain the elastic buckling behavior of carbon nanotubes by $\mathrm{Li}$ and $\mathrm{Chou}$ [13]. The results from this study indicate the improvement in the critical axial compressive load of a double-walled CNT (DWCNT) to that of a singlewalled CNT (SWCNT). Though all the previously mentioned studies suggest superior mechanical qualities of CNTs, the observed mechanical strengths $[14,15]$ are always lower than the theoretical predictions. This observation is attributed 
mainly due to the poor load transfer in between the layers of the MWCNT [16] and those of the interspatial layers in a CNT bundle [14]. We further need to note that the fabricated CNTs [17] are seldom perfectly straight in the laboratory conditions resulting in its nonideal nature. The effect of these curvatures on the elastic properties of CNTs with large aspect ratio can never be ignored [18]. In the first part of our present study, we report the elastic properties of a nonideal SWCNT array comprising long imperfectly straight SWCNTs [18] with a single bent along the tube axis. We find that the buckling characteristics are strongly affected by the interspatial distance of the SWCNT array due to the variations in arranging a bent orientation. Based on this understanding, we define a nonideal DWCNT in which the center core SWCNT is arranged at an offset distance to the outer SWCNT. MD simulation technique is employed to investigate the buckling characteristics of the nonideal DWCNT. The results clearly indicate that the resistance to buckling in a DWCNT arises mainly from the interlayer van der Waals interactions which are strongly influenced by the interlayer spacing. It should further be noted that close spacing of the adjacent CNT layers in a nonideal MWCNT introduces cross-links [19] which enhance the interwall shear strength. Liew et al. [20] performed buckling analysis of abnormal MWCNT by deploying a multishell continuum model with a refined van der Waal force model. Their investigations revealed that the effect of the van der Waals interaction is more significant for abnormal MWCNT than for normal MWCNT. Additionally, Song et al. [21] performed numerical simulation analysis to investigate the torsional responses of an abnormal MWCNT. They found that the critical torsional moments of DWCNTs are considerably enhanced due to the abnormal interlayer spacing of the CNT. Their results were significant due to the fact that the critical torsional moment does not always increase with decrease in the interlayer distance of DWCNTs. Zhang et al. [22] proposed a computer simulation model of novel DWCNTs with an interlayer distance of less than $3.4 \AA$ with improved compressive stability. The MD studies on DWCNTs with abnormal interlayer distances by Song and Zhi [23] further confirmed that the small interlayer spacing provides an effective channel for load transfer of outer and inner tubes and permits mechanical participation of two walls. Therefore, an optimum design of the interlayer spacing in a DWCNT will result in a superior mechanical performance. We would however like to remark that the current study aims to propose only a numerical model of nonideal DWCNT, and its fabrication method still needs to be investigated. The following section presents the computational model employed in our current work. The studies on the compressive characteristics of SWCNT array are described next followed by our research on the DWCNT. Finally we summarized our findings in the conclusion section.

\section{Numerical Simulation and Model}

In the current study, the interatomic forces that act in between the covalently bonded carbon atoms of the CNT were computed using the Brenner's second generation reactive empirical bond order function [25]. The long range Lennard-Jones 12-6 potential [26] is further deployed to account for the non-bonded interactions in an SWCNT array and the DWCNT. The mathematical representation of the previously mentioned potentials is given as

$$
E_{\mathrm{REBO}}=V_{R}\left(r_{i j}\right)-b_{i j} V_{A}\left(r_{i j}\right),
$$

where the repulsive and attractive pair terms are given by $V_{R}$ and $V_{A}$, respectively. The $b_{i j}$ term is used to include the reactive empirical bond order between the atoms. And the Lennard-Jones (12-6) potential is given as

$$
E_{\mathrm{LJ}}=4 \varepsilon\left[\left(\frac{\sigma}{r_{i j}}\right)^{12}-\left(\frac{\sigma}{r_{i j}}\right)^{6}\right],
$$

where $\varepsilon=4.55 \mathrm{meV}$ which is the well depth parameter and $\sigma=3.4 \AA$ is the collision diameter between two atoms [27].

The complete form of the potential employed is therefore given by

$$
E_{\mathrm{CNT}}=E_{\mathrm{REBO}}+E_{\mathrm{vdW}} .
$$

The contribution due to the van der Waals interaction $\left(E_{\mathrm{vdW}}\right)$ is accounted for when the covalent potential given by $E_{\mathrm{REBO}}$ becomes zero for bond lengths exceeding $2.0 \AA . E_{\mathrm{vdW}}$ is therefore defined as [28]

$$
\begin{aligned}
& E_{\mathrm{VdW}} \\
& \quad= \begin{cases}0, & r_{i j} \leq 2.0 \AA, \\
c_{3, k}\left(r_{i j}-r_{k}\right)^{3}+c_{2, k}\left(r_{i j}-r_{k}\right)^{2}, & 2.0 \AA<r_{i j} \leq 3.2 \AA, \\
E_{\mathrm{LJ}}\left(r_{i j}\right), & 3.2 \AA<r_{i j} \leq 10.0 \AA,\end{cases}
\end{aligned}
$$

where $c_{n, k}$ are the cubic spline coefficients for computation of the potential [28] when it turns repulsive when $2.0 \AA<r_{i j} \leq$ $3.2 \AA$.

The mechanical properties of the nonideal SWCNT and DWCNT were investigated using MD simulation. The simulation procedure consists of defining the initial positions of atoms after which it is subjected to relaxation using the potential model represented above to obtain the relaxed energy positions using conjugate gradient technique in an NVE ensemble. The carbon atoms at both ends of the system are fixed not to move in the plane normal to the tube axis and subjected to a finite inward displacement. The remaining atoms are subjected to relaxation after every 1000 time steps where the data is extracted. The procedure is repeated until the system buckles completely. This simulation consists of a total of 200,000 time steps with each time step equivalent to 1 fs.

\section{Studies on SWCNT Array}

3.1. Description of the Array System. It is evident from our previous study [18] that the critical compressive strain in a nonideal imperfectly straight SWCNT will depend strongly 


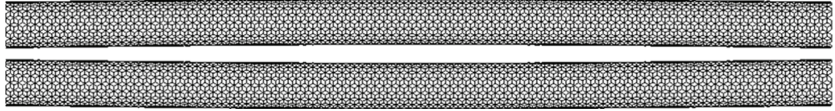

(a)

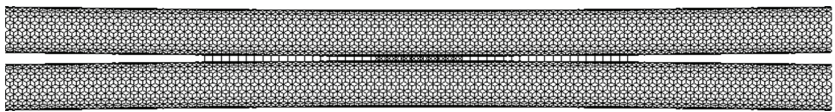

(b)

Figure 1: System of two "C" shaped CNTs arranged to form (a) a convex array and (b) a concave array.

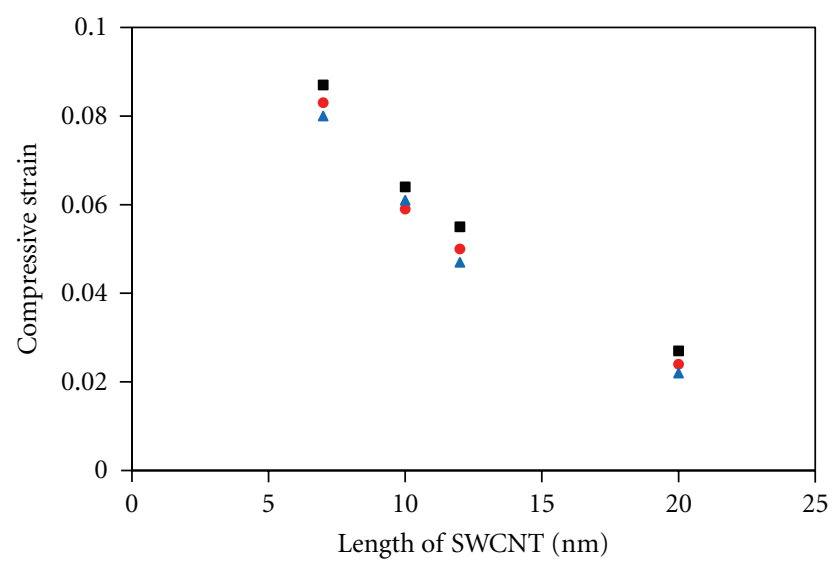

- Perfectly straight SWCNT

- "C" shaped SWCNT

$\Delta$ Reference $[24]$

Figure 2: The variation in the compressive strain of the $(16,0)$ SWCNT and its comparison with the virtual experiment results of [24].

on the number of bends present along the axis of CNT. As an extension, we have investigated the effect of bend orientation on the buckling properties of nonideal CNT array system comprising imperfectly straight SWCNTs. Using the same naming convention as in [18], we define an imperfectly straight $(10,10)$ SWCNT with a single bent along its tube axis as a "C" shaped SWCNT with length $l=245 \AA$. Two sets of SWCNT array configurations are deployed and are hence described.

(1) Array comprising two "C" shaped SWCNTs in which the bends face away from each other to form a convex system (referred to as convex array).

(2) Array of two "C" shaped SWCNTs in which the bends face towards each other to form a concave system (represented as concave array).

The two "C" shaped SWCNTs are arranged with an interspacial gap of $3.4 \AA$ along the $x$-axis, with the array configuration illustrated in Figure 1.

The buckling characteristic of an SWCNT is considered first in our study. For this purpose, we chose a $(16,0)$ SWCNT of "C" shaped and perfectly straight pristine configurations of various lengths. The variation in the compressive

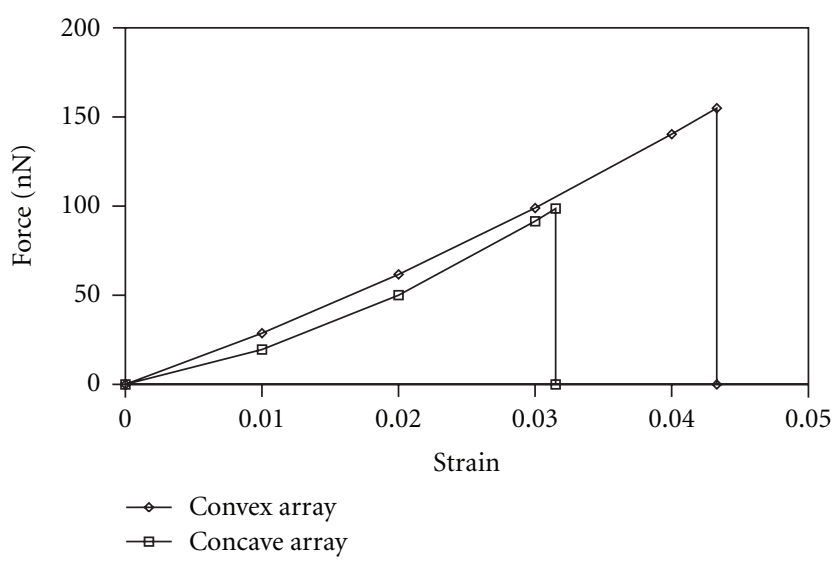

FIGURE 3: Force-strain plot of concave and convex SWCNT array.
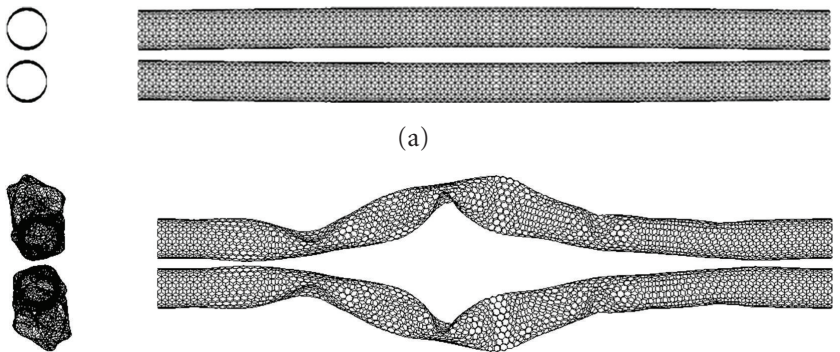

(a)

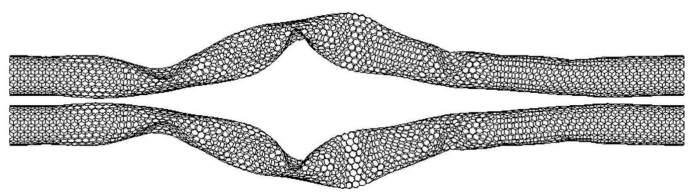

(b)

Figure 4: Morphological structural deformation of the convex array at (a) strain $\varepsilon=0.00$ and (b) at $\varepsilon=0.0433$.

strain of the SWCNT with length is depicted in Figure 2. We also compared the results with the virtual experiment results of the $(16,0)$ SWCNT performed by Sears and Batra [24]. As described in [24], the virtual experiment follows a procedure that closely mimics traditional macroscopic material tests. It can be seen from Figure 2 that the variation in the compressive strain reduces due to the inclusion of a curvature along the tube axis. Additionally, this procedure also helps us to validate our simulation model as described in Section 2.

We commence our study on nonideal CNT array comprising imperfectly straight SWCNTs by investigating the buckling characteristics of the convex array. The force-strain plot of the array system deployed in the study is given in Figure 3. For the array in which the two "C" shaped SWCNTs form a convex arrangement, the critical buckling load that the system can withstand is $P_{\mathrm{cr}}=150 \times 10^{-9} \mathrm{~N}$, and the corresponding critical strain value is $\varepsilon=0.0433$. This is certainly a much improved performance over the concaved system which buckles at $\varepsilon=0.0315$. The load-carrying capacity of the system is $P_{\mathrm{cr}}=97 \times 10^{-9} \mathrm{~N}$, a reduction by about $33 \%$. The morphological structural deformation of the convex and concave array is depicted in Figures 4 and 5, respectively.

3.2. Discussion. The simulation results of the array of imperfectly straight SWCNTs reveal that the buckling characteristics show a marked variation with which the bends 

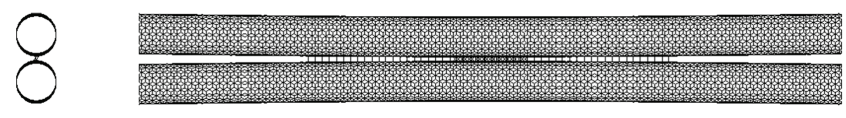

(a)
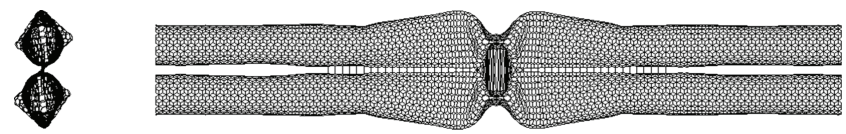

(b)

FIGURE 5: Morphological structural deformation of the concave array at (a) strain $\varepsilon=0.00$ and (b) at $\varepsilon=0.0315$.
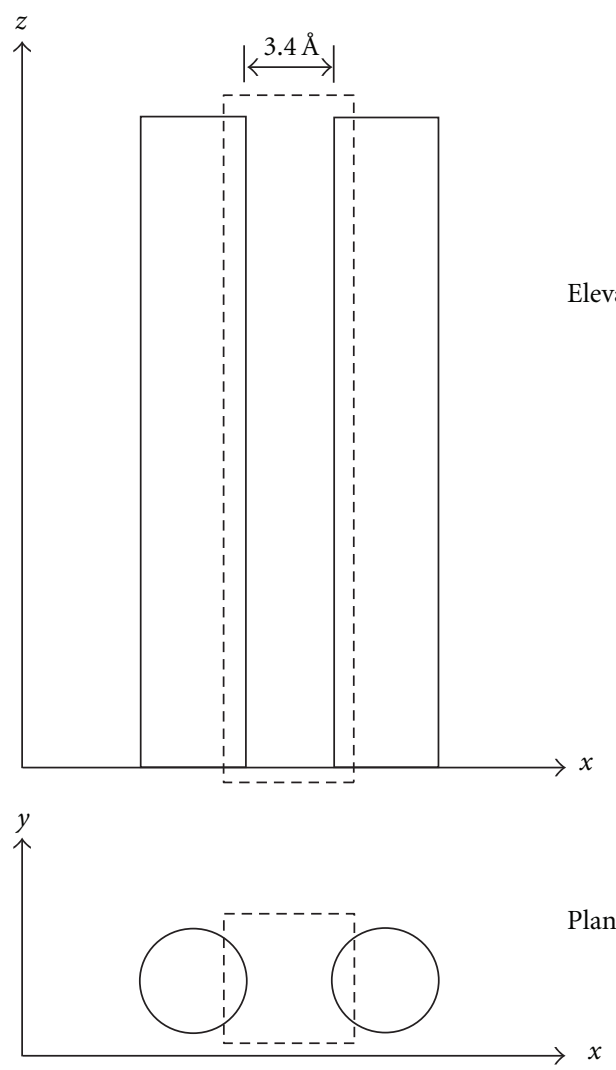

Figure 6: Measurement of the inter-tube van der Waals force component. The force components due to the van der Waals interaction are measured in between the atoms from adjacent facets of the SWCNTs in an array. This region is marked by the dashed rectangle. The interfacial spacing is at $3.4 \AA$ along the $x$ axis.

are arranged. A quantitative explanation to the buckling behavior of the SWCNT array is explained by making use of the intertube van der Waals forces in the array system. This is due to the fact that in a system of CNT array or MWCNT, van der Waals interaction becomes a decisive component $[18,29,30]$ that decides the mode of buckling.

The buckling load of a CNT array is increased due to the intertube van der Waals interaction by about $3.8 \sim 5.2 \%$, as reported in [29]. This interactive force is measured by considering the reaction in between the atoms that belong to the adjacent opposite walls of the two "C" shaped SWCNTs that face each other as indicated by the region enclosed within the dashed rectangle as shown in Figure 6. The intertube van der Waals force has three mutually perpendicular components directed along the $x, y$, and $z$ direction respectively. We have considered only the $x$ component of the force because the tube spacing is across the $x$-axis, and the force components along the $y$ and $z$ axis are negligible.

Figures 7 and 8 show the macroscopic view of the central interspacial region that separates the two "C" shaped CNTs for both the array systems considered in our study. We observe from the figure that for a convex array, due to the bends facing opposite direction, the interspacial distance will be greater than $3.4 \AA$. Similarly, for the concave array the distance will be lesser than $3.4 \AA$ due to the bends facing each other. This variation in distance affects the interatomic distance in between the two adjacent facets as explained earlier, which in turn affects the inter-tube van der Waal forces. The $x$ component of the inter-tube van der Waals force is denoted by $F_{x}^{\mathrm{vdW}}$ in our study. The relationship between $F_{x}^{\mathrm{vdW}}$ and the strain is depicted in Figure 9 for the convex and concave array systems. It can be seen from Figure 9 that the value of $F_{x}^{\mathrm{vdW}}$ for a convex array is always higher than that of the concave array. This shows a strong correlation between the van der Waals force and the interspacial distance that separates the two "C" shaped SWCNTs. We can also observe that near the point of critical strain, a steep drop in $F_{x}^{\mathrm{vdW}}$ is observed which indicates that the tube has buckled. It is evident that with the decrease in the van der Waals force, the strengthening effect of the SWCNT array becomes weaker, and it initiates buckling at that particular value of critical strain. Additionally, we also observe that the concave nature of arrangement in the CNT array results in the formation of inter-tube bonds to be formed. These inter-tube bonds are formed mainly due to the reduction in the $\mathrm{C}-\mathrm{C}$ bond distance resulting in the formation of more $s p^{3}$ configuration bonds. These $s p^{3}$ bonds increase the strain energy of the concave array system (Figure 10) when compared to the convex array. This can be observed by the strain energy increase corresponding to the concave array system in Figure 10 for compressive strain value approaching the plastic limit. This increase in the internal strain energy causes the rapid failure under the application of compressive forces.

\section{Studies on DWCNT}

4.1. Description of the DWCNT System. It is evident from Section 3 that the variation in the interspacial distance strongly influences the nonbonded interaction. This in turn affects the elastic properties of a nonbonded CNT system. In this section, we aim to study the variation in the buckling characteristics of a DWCNT by varying the interlayer distance in between the two SWCNTs and hence resulting in a nonideal DWCNT. The DWCNT used in our study consists of a $(10,10)$ SWCNT nested inside a $(15,15)$ SWCNT of a shorter length $l=98.5 \AA$. Due to the shorter length, we assumed that the DWCNT is perfectly straight, that is, pristine. A schematic of the DWCNT system is given in Figure 11 where the interlayer region between the two 


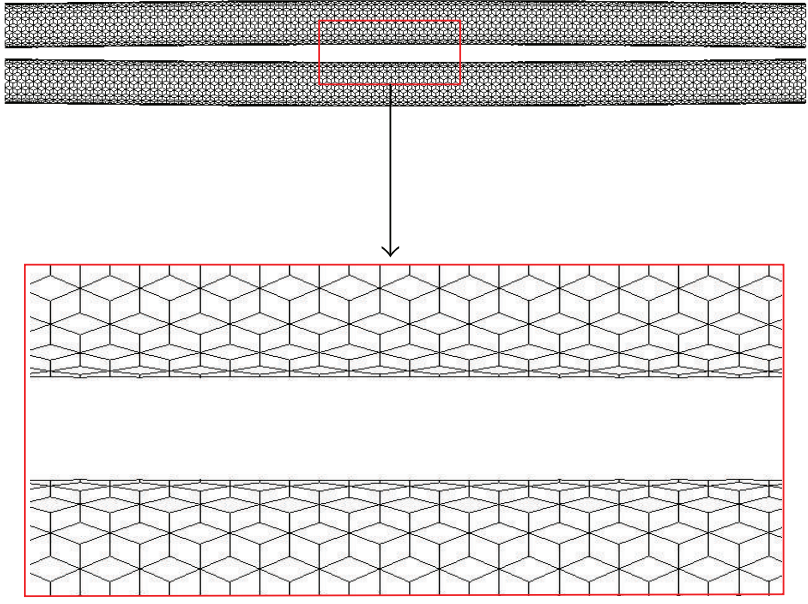

FIGURE 7: Illustration of the inter-tube spacing in the convex array. Even though the inter-spatial distance in between the tubes at both ends of the convex array is kept at $3.4 \AA$, the corresponding distance at the central region is greater than $3.4 \AA$.

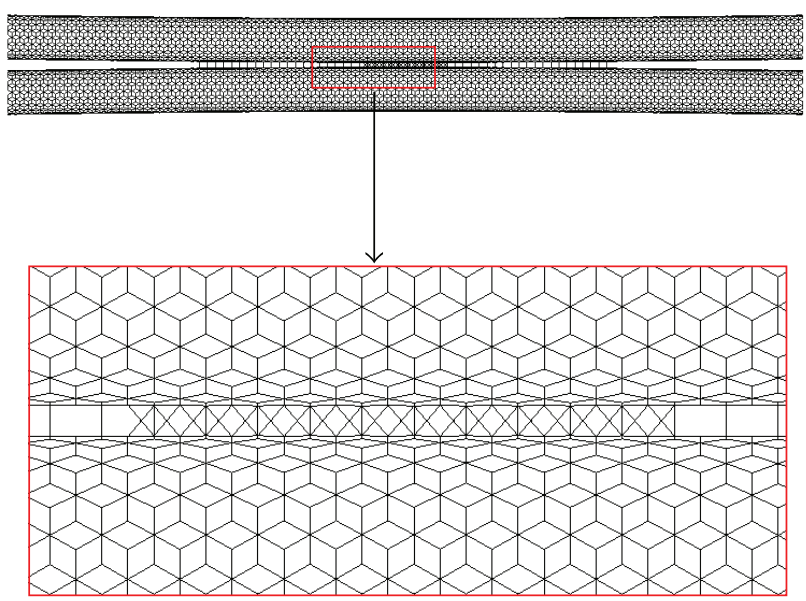

FIGURE 8: Illustration of the inter-tube spacing in a concave array. Atoms near the central region of a nonideal concave SWCNT array are more closely spaced resulting in the formation of C-C bonds.

SWCNTs in the top half is represented as region 1 and those in the bottom half as region 2. Three different classes of DWCNTs are deployed in our study, namely, classes "A," "B" and " $\mathrm{C}$." The classification is based on the interlayer distance in region 1 and region 2 as given in Table 1 . It should be noted from Table 1 that class "B" is an ideal DWCNT while classes " $\mathrm{A}$ " and " $\mathrm{C}$ " are categorized as nonideal DWCNTs with nonconcentric constituent SWCNTs. The class "B" DWCNT is equilibrated using conjugate gradient technique in an NVE ensemble. It should be noted that energy relaxation of class " $\mathrm{A}$ " and class " $\mathrm{C}$ " DWCNT will result in a concentric arrangement (i.e., class "B"). Hence to maintain the non-concentricity of class " $\mathrm{A}$ " and class " $\mathrm{C}$ " DWCNT structure, we did not perform any energy minimization operations and these two classes of DWCNTs are left as a prestressed structure before they were subjected to compression.

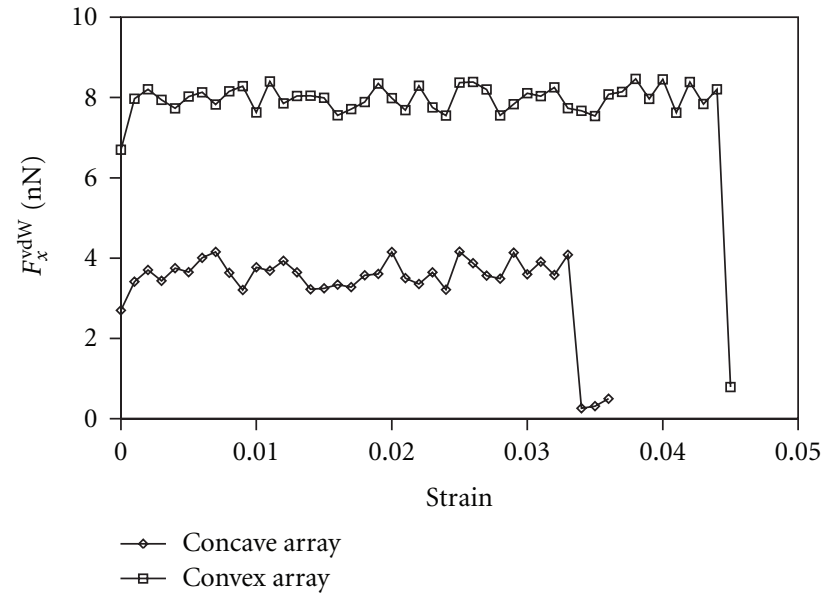

FIGURE 9: The inter-tube van der Waals force versus the strain acting across the inter-tube spacing between the atoms in the $x$-direction from adjacent facets of the convex and concave array systems.

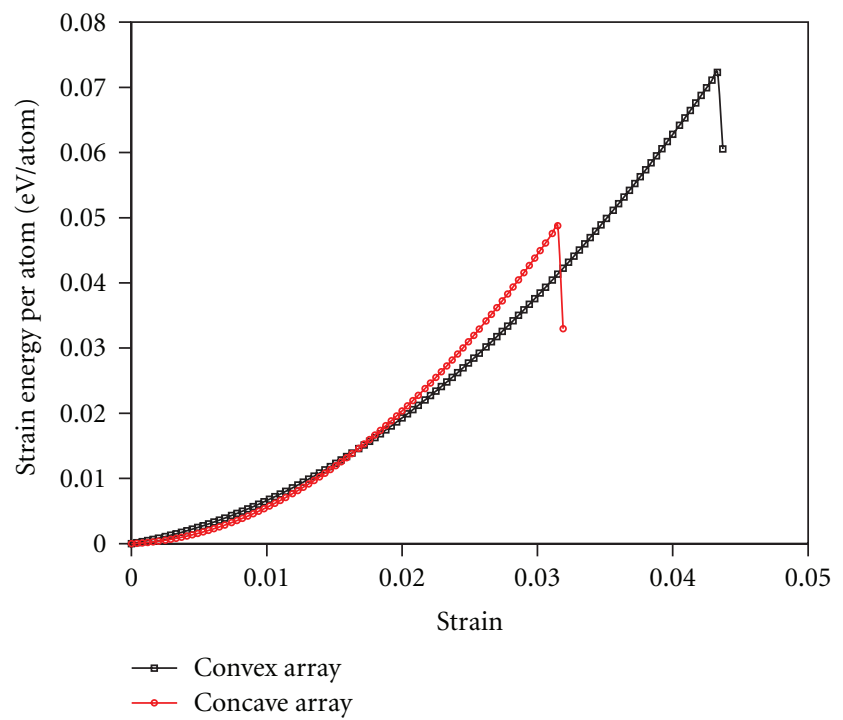

FIGURE 10: Strain energy per atom plot of the concave and convex array of $(10,10)$ SWCNT. Formation of inter-tube bonds in a concave array increases the strain energy of the concave array SWCNT system.

TABle 1: Description of the classes of DWCNTs considered in our study classified based on the inter-layer distance in region 1 and region 2 as described in Figure 11.

\begin{tabular}{lcc}
\hline \multirow{2}{*}{ DWCNT } & \multicolumn{2}{c}{ Interlayer Distance $(\AA)$} \\
& Region 1 & Region 2 \\
\hline Class A & 4.86 & 1.92 \\
Class B & 3.38 & 3.39 \\
Class C & 3.78 & 3.00 \\
\hline
\end{tabular}

4.2. Results. The buckling characteristic of non-concentric DWCNTs is described by making use of the force-strain plot as depicted in Figure 12. From this plot we observe that the normal class "B" DWCNT is able to resist the compression 


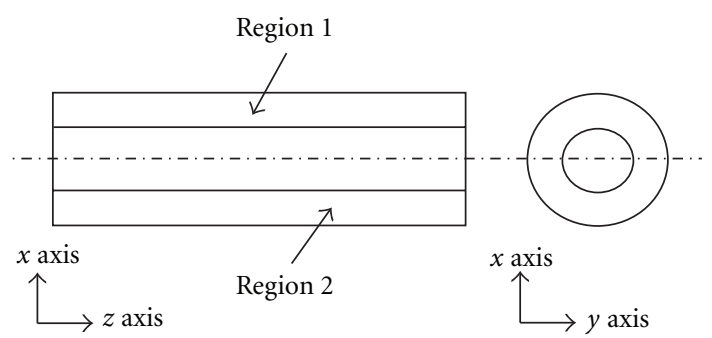

FIGURE 11: Schematic view of a DWCNT used in our study with the interlayer regions marked as region 1 and region 2 .

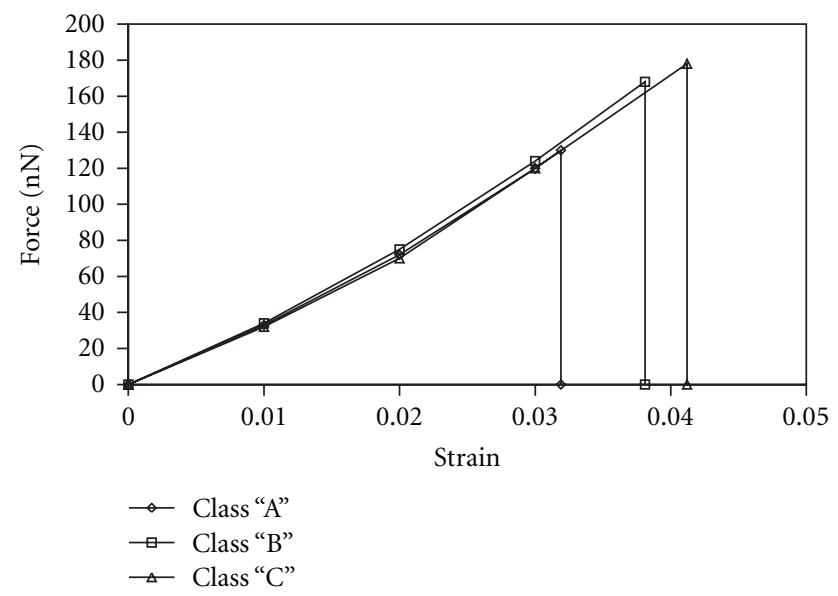

FIGURE 12: Force-strain plot of the DWCNT classes used in our study subjected to compression.

forces for an extended period compared to that of the class "A" DWCNT with abnormal interlayer distance. The critical buckling load of the class "B" DWCNT is $168 \mathrm{nN}$ and its critical buckling strain is $\varepsilon=0.0381$. Superior elastic properties are observed in a class " $\mathrm{C}$ " DWCNT system which buckles at a critical buckling strain of $\varepsilon=0.0412$ and critical buckling load of $178 \mathrm{nN}$. The class "A" DWCNT exhibits the least resistance to the buckling when compared to class " $\mathrm{B}$ " and class "C" DWCNT systems and it buckles at a critical strain of $\varepsilon=0.0319$ with the critical buckling load of $130 \mathrm{nN}$. The morphological variations of the three classes of DWCNT under compression are depicted in Figure 13.

\subsection{Discussion}

4.3.1. Inter-Tube van der Waals Interaction. The variation in elastic properties of the three classes of DWCNTs can once again be explained by the non-bonded interactions that act in between the adjacent layers of the DWCNT. Since in our current study, the nesting of DWCNT is oriented along the $x-y$ plane (Figure 11), we have considered the resultant of the $x$ and $y$ components of the van der Waals force that act in between the two component SWCNTs. Mathematically this resultant force is represented as $F_{x y}^{\mathrm{vdW}}$. It is evident from the plot (Figure 14) that the magnitude of the inter-tube van der Waals force for the class "C" DWCNT is the highest which results in its superior performance under compression when
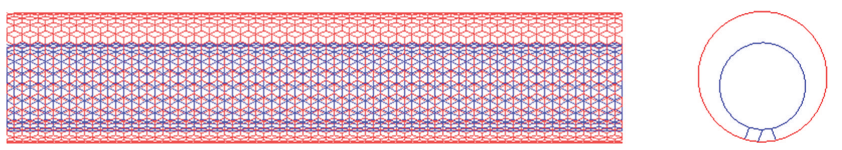

(i)
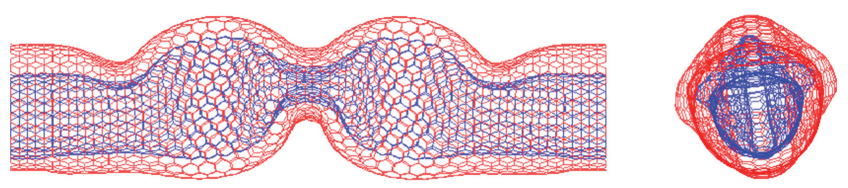

(ii)

(a)
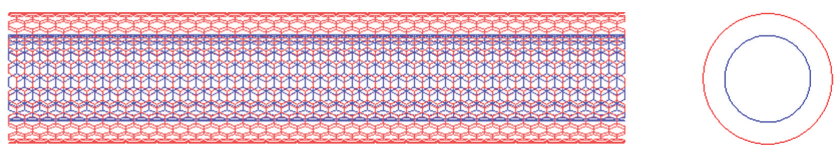

(i)
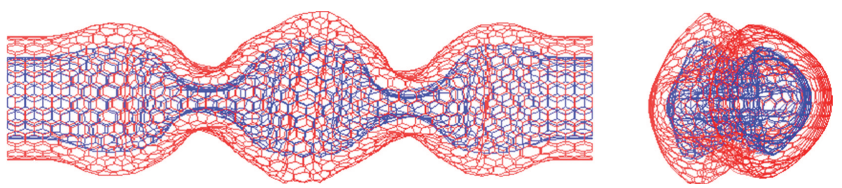

(ii)

(b)
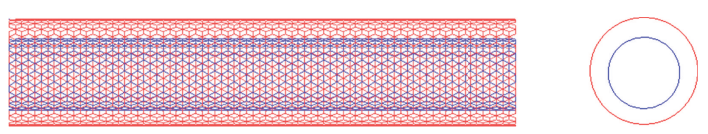

(i)

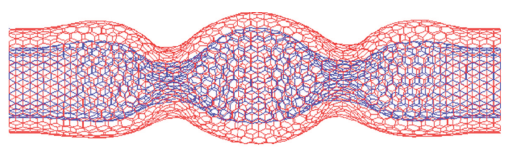

(ii)

(c)

FIGURE 13: Morphological changes of the three classes of DWCNTs under buckling. (a) A class "A" DWCNT at (i) strain, $\varepsilon=0.0$, (ii) strain, $\varepsilon=0.0319$, (b) A class "B" DWCNT at (i) strain, $\varepsilon=0.0$, (ii) strain, $\varepsilon=0.0381$ and (c) A class "C" DWCNT at (i) strain, $\varepsilon=0.0$, (ii) strain, $\varepsilon=0.0412$.

compared to the class "A" and class "B" counterparts. Similar to what we observe in a CNT array system, a steep decrease in van der Waals forces indicate the onset of buckling. Resistance to buckling is affected by the stability of these nonbonded forces during the period of elasticity after which the DWCNT will buckle when the inter-tube van der Waals force becomes zero.

4.3.2. Interwall Separation. It is also useful to consider the effect of interwall spacing to describe the compressive behavior of the non-concentric DWCNTs. Since from Figure 11 the offset alignment is oriented across the $x$ direction, let us focus on the $x$ component of the inter-tube van der Waals forces that act in region 1 and region 2. These force components are denoted as $\left[F_{x}^{\mathrm{vdW}}\right]_{\text {region1 }}$ and $\left[F_{x}^{\mathrm{vdW}}\right]_{\text {region2 } 2}$, and the corresponding plots are given in Figures 15 and 16, 


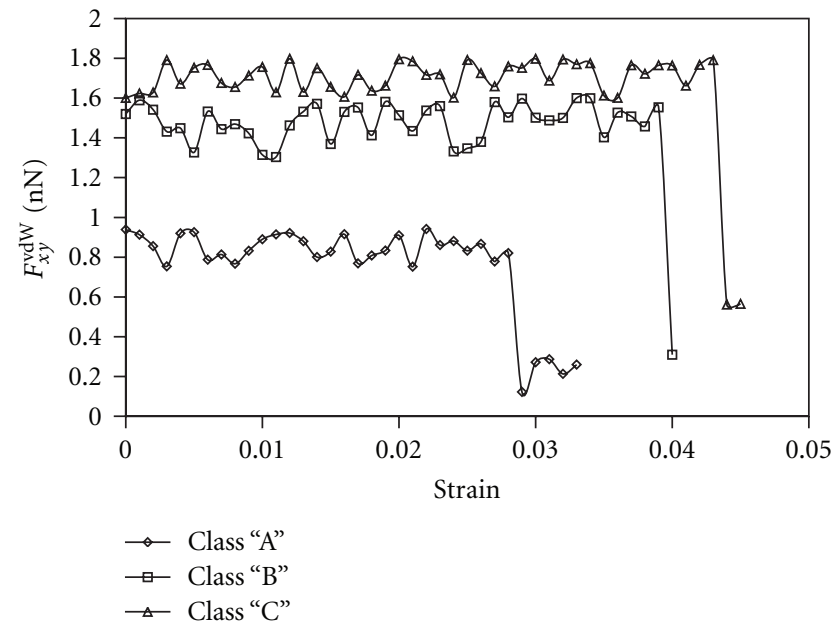

Figure 14: The resultant of the $x$ and $y$ components of the intertube van der Waals force acting across the inter-tube spacing between the atoms from adjacent layers of the inner $(10,10)$ SWCNT and the outer $(15,15)$ SWCNT.

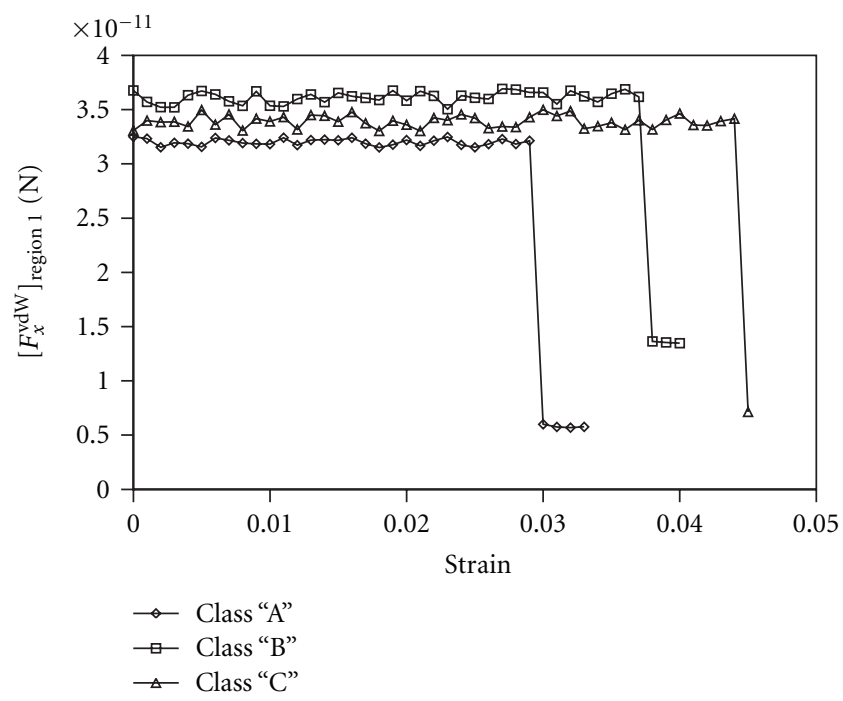

FIGURE 15: The $x$ component of the inter-tube van der Waals force acting across the atoms from adjacent layers of the inner $(10,10)$ SWCNT and the outer $(15,15)$ SWCNT in the region 1 for the three classes of DWCNT considered in our study.

respectively. We note from (4) that the interlayer spacing in region 1 falls in between 3.2 , and $10.0 \AA$, and the governing interaction is mainly due to the $E_{\mathrm{LJ}}\left(r_{i j}\right)$ term and this interactive potential decreases with increasing interatomic distance. Since the inter-layer spacing for the class "B" DWCNT in region 1 is the lowest for all three classes of DWCNT which is at about $3.4 \AA$, it exhibits a higher value of interactive van der Waals force in region 1.

It is interesting to observe the interaction forces in region 2 of all the three classes of DWCNT (Figure 16). The interlayer spacing of three classes of DWCNT in this region is distinct which will cause a functional variation in the interactive potential as described in (4). For a class "A"

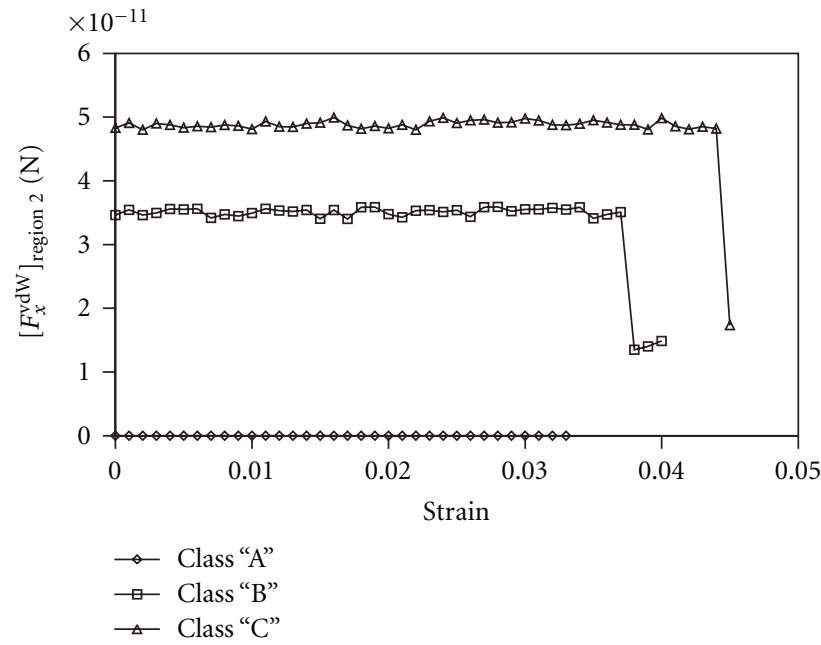

FIGURE 16: The $x$ component of the inter-tube van der Waals force acting across the atoms from adjacent layers of the inner $(10,10)$ SWCNT and the outer $(15,15)$ SWCNT in the region 2 for the three classes of DWCNT considered in our study.

DWCNT in which the interlayer separation is below $2.0 \AA$, the value of $\left[F_{x}^{\mathrm{vdW}}\right]_{\text {region2 }}$ is zero. Since the resistance to buckling in a DWCNT is caused by the intertube van der Waals interaction, we can conclude that there is effectively no contribution to the resistive forces from the region 2 of the class "A" DWCNT which aids in its premature buckling. Furthermore, superior resistance to the buckling of a class " $\mathrm{C}$ " DWCNT is offered by the interaction forces from the region 2 which are spaced at $3.0 \AA$ as described by the cubic spline polynomial in (4). A nominal performance is expected from the normal class " $\mathrm{B}$ " DWCNT where the interaction among the layers spaced at $3.4 \AA$ is determined by the $E_{\mathrm{LJ}}\left(r_{i j}\right)$ term.

4.3.3. Strain Energy. Figure 17 shows the plot of strain energy per atom of the inside $(10,10)$ SWCNT in all three classes of DWCNT considered in our study. We can observe that until all three DWCNTs collapse due to compressive forces, the magnitude of strain energy per atom for the class " $\mathrm{C}$ " DWCNT is always smaller than the other two classes, namely, "A" and "B". Furthermore, at the point of critical buckling strain, there is a sudden increase in strain energy for class " $\mathrm{B}$ " and class " $\mathrm{C}$ " DWCNT. This increase in strain energy is due to the spontaneous increase in potential energy of atoms in the DWCNT [6]. However, in the case of the class "A" DWCNT, a drop in strain energy is observed during buckling due to spontaneous plastic collapse [31]. This nature is due to the fact that the arrangement of class " $\mathrm{A}$ " DWCNT results in the formation of inter-tube bonds which are composed of $s p^{3}$ hybridized configuration. As explained in Section 3.2, we see that these inter-tube bonds play prominent role in increasing the strain energy of the class "A" DWCNT. Hence, we deduce from Figure 17 that application of compressive forces on a class "A" DWCNT will result in accelerated accumulation of strain energy that enhances the system instability leading to its premature failure. 


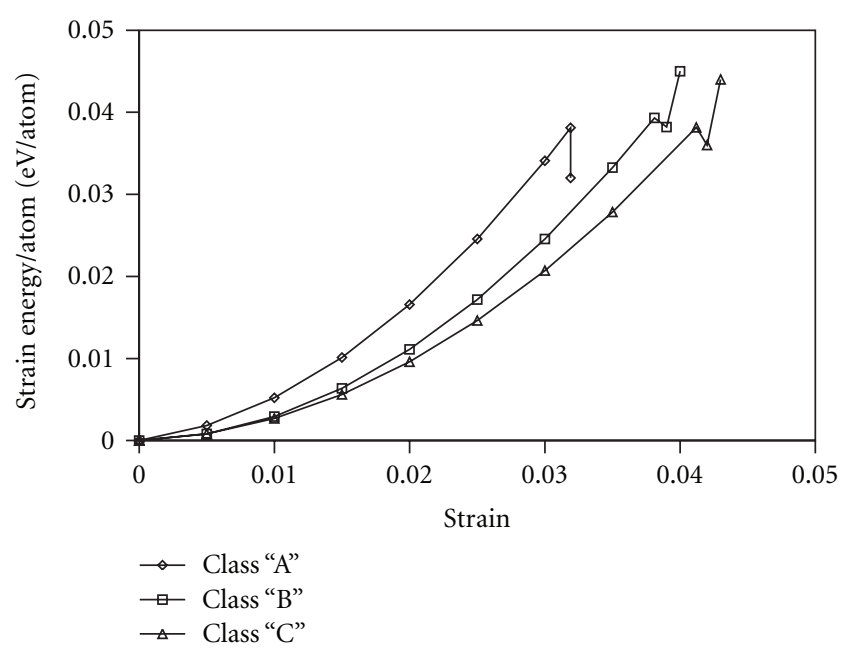

FIGURE 17: Plot of the strain energy per atom for the inside $(10,10)$ SWCNT in the three classes of DWCNT considered in our study.

\section{Conclusion}

We performed a theoretical investigation on the structural properties of the nonbonded CNT systems using molecular dynamics simulation technique. We constructed an array of two "C" shaped SWCNTs oriented in mutually opposite directions and reported a variation in the buckling characteristics. Our results show that these orientations affect the interspacial distance which in turn affects the nonbonded interactive forces in the adjacent facets of the CNT array. Resistance to buckling of an SWCNT array is caused due to the van der Waals forces which are explained quantitatively in our research. Similarly, our studies on the compressive characteristics of DWCNT also show the influence of the interlayer van der Waals forces to the compressive stability of the DWCNT. Suitable placement of the central SWCNT in a DWCNT system will result in enhanced mechanical performance as is evident from the buckling characteristics of the "class C" DWCNT. With recent developments in the fabrication process of carbon nanotubes, we hope that the findings obtained from this work may help in designing highperforming DWCNT and CNT array systems.

\section{References}

[1] E. T. Thostenson, Z. Ren, and T. W. Chou, "Advances in the science and technology of carbon nanotubes and their composites: a review," Composites Science and Technology, vol. 61, no. 13, pp. 1899-1912, 2001.

[2] A. Krishnan, E. Dujardin, T. W. Ebbesen, P. N. Yianilos, and M. M. J. Treacy, "Young's modulus of single-walled nanotubes," Physical Review B, vol. 58, no. 20, pp. 14013-14019, 1998.

[3] K. M. Liew, X. Q. He, and C. H. Wong, "On the study of elastic and plastic properties of multi-walled carbon nanotubes under axial tension using molecular dynamics simulation," Acta Materialia, vol. 52, no. 9, pp. 2521-2527, 2004.

[4] M. M. J. Treacy, T. W. Ebbesen, and J. M. Gibson, "Exceptionally high Young's modulus observed for individual carbon nanotubes," Nature, vol. 381, no. 6584, pp. 678-680, 1996.
[5] B. I. Yakobson, C. J. Brabec, and J. Bernholc, "Nanomechanics of carbon tubes: instabilities beyond linear response," Physical Review Letters, vol. 76, no. 14, pp. 2511-2514, 1996.

[6] K. M. Liew, C. H. Wong, X. Q. He, M. J. Tan, and S. A. Meguid, "Nanomechanics of single and multiwalled carbon nanotubes," Physical Review B, vol. 69, no. 11, Article ID 115429, 8 pages, 2004.

[7] J. Che, T. Çağin, and W. A. Goddard, "Studies of fullerenes and carbon nanotubes by an extended bond order potential," Nanotechnology, vol. 10, no. 3, pp. 263-268, 1999.

[8] C. Q. Ru, "Axially compressed buckling of a doublewalled carbon nanotube embedded in an elastic medium," Journal of the Mechanics and Physics of Solids, vol. 49, no. 6, pp. 12651279, 2001.

[9] C. Y. Wang, C. Q. Ru, and A. Mioduchowski, "Axially compressed buckling of pressured multiwall carbon nanotubes," International Journal of Solids and Structures, vol. 40, no. 15, pp. 3893-3911, 2003.

[10] F. M. Tong, C. Y. Wang, and S. Adhikari, "Axial buckling of multiwall carbon nanotubes with heterogeneous boundaries," Journal of Applied Physics, vol. 105, no. 9, Article ID 094325, 7 pages, 2009.

[11] W. B. Lu, J. Wu, X. Feng, K. C. Hwang, and Y. Huang, "Buckling analyses of double-wall carbon nanotubes: a shell theory based on the interatomic potential," Journal of Applied Mechanics, vol. 77, no. 6, Article ID 061016, 6 pages, 2010.

[12] X. Q. He, S. Kitipornchai, and K. M. Liew, "Buckling analysis of multi-walled carbon nanotubes: a continuum model accounting for van der Waals interaction," Journal of the Mechanics and Physics of Solids, vol. 53, no. 2, pp. 303-326, 2005.

[13] C. Y. Li and T. W. Chou, "Modeling of elastic buckling of carbon nanotubes by molecular structural mechanics approach," Mechanics of Materials, vol. 36, no. 11, pp. 10471055, 2004.

[14] M. F. Yu, B. S. Files, S. Arepalli, and R. S. Ruoff, “Tensile loading of ropes of single wall carbon nanotubes and their mechanical properties," Physical Review Letters, vol. 84, no. 24, pp. 5552-5555, 2000.

[15] M. F. Yu, O. Lourie, M. J. Dyer, K. Moloni, T. F. Kelly, and R. S. Ruoff, "Strength and breaking mechanism of multiwalled carbon nanotubes under tensile load," Science, vol. 287, no. 5453, pp. 637-640, 2000.

[16] J. Cumings and A. Zettl, "Low-friction nanoscale linear bearing realized from multiwall carbon nanotubes," Science, vol. 289, no. 5479, pp. 602-604, 2000.

[17] S. Iijima and T. Ichihashi, "Erratum: single-shell carbon nanotubes of 1-nm diameter (Nature (1993) 363 (603-605))," Nature, vol. 364, no. 6439, p. 737, 1993.

[18] C. H. Wong and V. Vijayaraghavan, "Nanomechanics of imperfectly straight single walled carbon nanotubes under axial compression by using molecular dynamics simulation," Computational Materials Science, vol. 53, no. 1, pp. 268-277, 2011.

[19] A. J. R. da Silva, A. Fazzio, and A. Antonelli, "Bundling up carbon nanotubes through wigner defects," Nano Letters, vol. 5, no. 6, pp. 1045-1049, 2005.

[20] K. M. Liew, J. B. Wang, X. Q. He, and H. W. Zhang, "Buckling analysis of abnormal multiwalled carbon nanotubes," Journal of Applied Physics, vol. 102, no. 5, Article ID 053511, 6 pages, 2007.

[21] H. Y. Song, L. F. Li, and F. Feng, "Torsional behaviour of carbon nanotubes with abnormal interlayer distances," Journal of Physics D, vol. 42, no. 5, Article ID 055414, 2009. 
[22] H. W. Zhang, L. Wang, and J. B. Wang, "Computer simulation of buckling behavior of double-walled carbon nanotubes with abnormal interlayer distances," Computational Materials Science, vol. 39, no. 3, pp. 664-672, 2007.

[23] H. Y. Song and X. W. Zha, "Molecular dynamics study of effects of intertube spacing on sliding behaviors of multiwalled carbon nanotube," Computational Materials Science, vol. 50, no. 3, pp. 971-974, 2011.

[24] A. Sears and R. C. Batra, "Buckling of multiwalled carbon nanotubes under axial compression," Physical Review B, vol. 73, no. 8, Article ID 085410, 11 pages, 2006.

[25] D. W. Brenner, O. A. Shenderova, J. A. Harrison, S. J. Stuart, B. Ni, and S. B. Sinnott, "A second-generation reactive empirical bond order (REBO) potential energy expression for hydrocarbons," Journal of Physics Condensed Matter, vol. 14, no. 4, pp. 783-802, 2002.

[26] L. E. Lennard-Jones, "On the determination of molecular fields. I. From the variation of the viscosity of a gas with temperature," Proceedings of the Royal Society A, vol. 106, no. 738, pp. 441-463, 1924.

[27] A. K. Rappé, C. J. Casewit, K. S. Colwell, W. A. Goddard, and W. M. Skiff, "UFF, a full periodic table force field for molecular mechanics and molecular dynamics simulations," Journal of the American Chemical Society, vol. 114, no. 25, pp. 1002410035, 1992.

[28] Z. G. Mao, A. Garg, and S. B. Sinnott, "Molecular dynamics simulations of the filling and decorating of carbon nanotubules," Nanotechnology, vol. 10, no. 3, pp. 273-277, 1999.

[29] K. M. Liew, C. H. Wong, and M. J. Tan, "Buckling properties of carbon nanotube bundles," Applied Physics Letters, vol. 87, no. 4, Article ID 041901, 3 pages, 2005.

[30] Y. Y. Jiang, W. Zhou, T. Kim, Y. Huang, and J. M. Zuo, "Measurement of radial deformation of single-wall carbon nanotubes induced by intertube van der Waals forces," Physical Review B, vol. 77, no. 15, Article ID 153405, 4 pages, 2008.

[31] O. Lourie and H. D. Wagner, "Transmission electron microscopy observations of fracture of single-wall carbon nanotubes under axial tension," Applied Physics Letters, vol. 73, no. 24, pp. 3527-3529, 1998. 

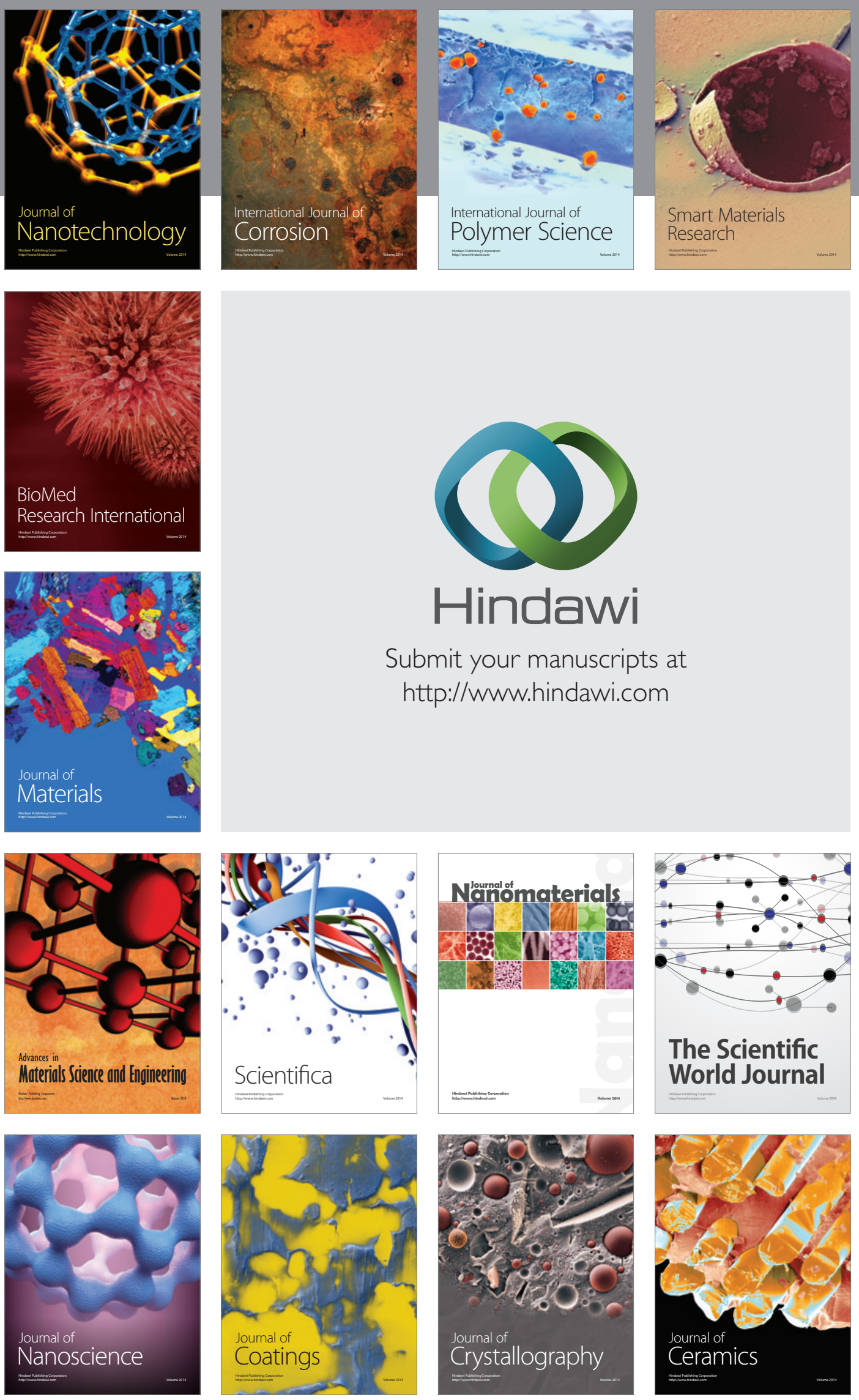

The Scientific World Journal

Submit your manuscripts at

http://www.hindawi.com

\section{World Journal}

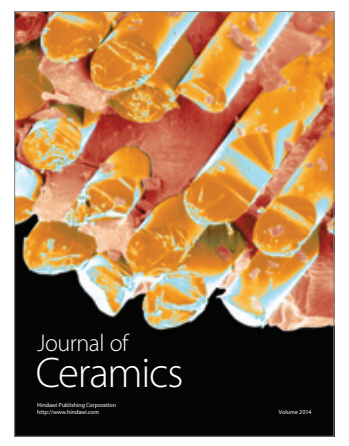

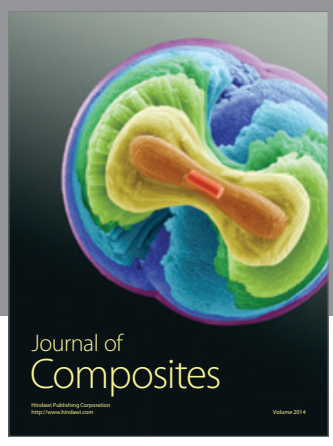
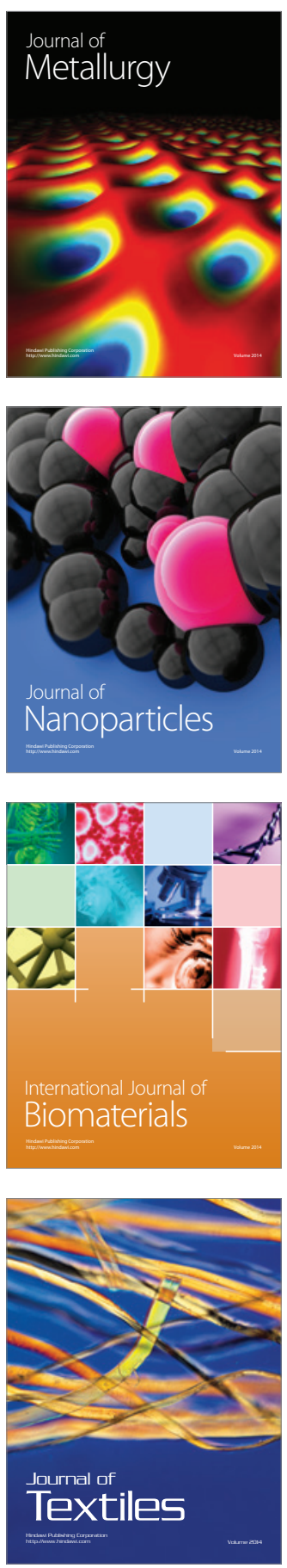